\title{
Hyaluronan is not just a goo!
}

\author{
Bryan P. Toole
}

See related article,

pages $349-360$.

Department of Anatomy and Cellular Biology, Tufts University School of Medicine, Boston,

Massachusetts 02111, USA. Phone: (617) 636-6659; Fax: (617) 636-0380; E-mail: bryan.toole@tufts.edu.

Long, long ago, when hyaluronan (HA; also termed hyaluronic acid or hyaluronate) was just a goo, a trickle of papers emerged suggesting that it might also play an important role during embryonic development, possibly creating hydrated pathways that facilitate cell movement (reviewed in ref. 1). Among these early publications were studies by Roger Markwald's group indicating that endothelial cells migrated into an HA-rich ECM during cardiac cushion development (2) and that HA might influence this process (3). In this issue of the JCI, Camenisch et al. (4) describe aberrant development of the cardiovascular system in mice in which one of the three known HA synthase genes, Has2, is knocked out. This study provides final proof that $\mathrm{HA}$ is indeed essential for heart development and highlights two probable molecular functions of HA.

HA, a polymer of glucuronic acid alternating with $N$-acetyl glucosamine, is a simple but unusual polysaccharide. It is a member of the glycosaminoglycan (GAG) family, but it differs in many ways from other GAGs. First, it is huge, usually with a molecular weight between $10^{3}$ and $10^{4} \mathrm{kDa}$ and an extended length of 2-25 $\mu \mathrm{m}$. Second, unlike other GAGs, HA contains no sulfate groups or epimerized uronic acid residues. Third, and central to the study described in this issue of the JCI (4), the mechanism of synthesis of HA is unique - HA is made at the inner side of the plasma membrane rather than in the Golgi apparatus; it is most likely elongated at the reducing rather than the nonreducing terminus during synthesis; and it is not covalently linked to a protein backbone during synthesis. After many years of frustration, characterization of the enzymes responsible for HA synthesis, i.e., the HA synthases (Has's), has progressed rapidly over the past several years (reviewed in refs. 5, 6). The first of these enzymes to be cloned and characterized was from pathogenic bacteria that produce protective capsules of HA that enhance their virulence. An operon involved in HA synthesis was characterized in group A Streptococcus, and the Has gene was cloned by transposon interruption of this operon in Streptococcus pyogenes (7). After expression, the encoded protein was shown to synthesize high-molecular weight HA, thus establishing that a single protein, encoded by the Has gene, is responsible for both of the glycosyltransferase activities required for synthesis of HA (8). Several groups have since succeeded in cloning and characterizing three separate genes for

\section{Hyaluronan, is a simple but unusual polysaccharide.}

vertebrate HA synthases: Has1, Has2, and $\operatorname{Has} 3(5,6)$. Camenisch et al. (4) now describe the production of Has $2^{-/-}$mice, which, they show, have serious cardiovascular defects and die during mid-gestation.

Has $2^{-/}$mouse embryos at stage E9.5 of development contain virtually no HA. At this stage they exhibit multiple defects, including yolk sac, vasculature, and heart abnormalities. In particular, the cardiac jelly and cardiac cushions, which normally give rise to valves and other structures, fail to form. At the tissue level, ECMs are more compact than normal, and the organization of various ECM components is altered. Part of the process of cardiac cushion remodeling involves transformation of endothelium to mesenchymal cells that migrate into the HA-rich cardiac jelly. To test for a possible role for HA in this transformation and migration, Camenisch et al. cultured developing cushion tissue explants on collagen gels and observed that endothelial-mesenchymal transformation and cell migration occur when wild-type tissue is used, but not when Has $2^{-/-}$tissue is used (4). Transformation and migration are rescued when the Has2 $2^{-/}$tissue expresses an Has2 cDNA or is treated with nanogram amounts of exogenous HA. Finally, the authors show that, although HA's effect on migration is apparently Ras-independent, HAmediated rescue of transformation can be mimicked by transfecting cells with constitutively active Ras and can be inhibited by a dominant-negative Ras cDNA. These results point toward at least two functions for HA. The first is in formation of a highly hydrated, expanded ECM, such as is found in the cardiac jelly. The second is in facilitating cell behavior, such as occurs during epithelial-mesenchymal transformation and migration of cushion endothelium.

The molecular functions of HA fall into three partially overlapping categories (reviewed in refs. 9, 10): First, HA occupies an enormous hydrodynamic domain that greatly influences the hydration and physical properties of tissues. Second, it interacts with other ECM macromolecules, including the proteoglycans aggrecan and versican; these interactions are essential to the structure and assembly of several tissues. Finally, HA interacts with cell surface receptors, notably CD44, and thereby influences cell behavior. The ability of HA to form a pericellular coat - a unique environment in which many cell types reside - illustrates the interrelation of these three functions. Formation of pericellular coats depends on the large hydrodynamic domain occupied by $\mathrm{HA}$, its interaction with extracellular proteoglycans, and its interaction with the cell surface. The data of Camenisch et al. (4) support the importance of the hydrodynamic properties of HA in expansion of the matrix in cardiac cushions, and the likelihood that interactions with ver- 
sican are critical in the structure of this matrix. Perhaps more unexpected are the requirement of HA in endothelial cell transformation and migration and the relationship of HA-cell interactions to Ras signaling.

Recent studies have made it increasingly clear that HA-cell interactions affect not only cell movement (11-13), as seen here, but also invasive behavior (14) and malignant transformation $(15,16)$. Interaction of HA with the HA receptor CD44, which accounts for some of this effect, stimulates intracellular signaling through $\operatorname{Rac}(11,12)$, phosphoinositide 3 '-kinase (17), and Ras (18) in various cell types in culture. The work of Camenisch et al. (4) establishes the importance of one such HAdependent pathway in embryonic development. Similar interactions are likely to be of paramount importance in cancer as well (14-17).

1. Toole, B.P. 1981. Glycosaminoglycans in morphogenesis. In Cell biology of extracellular matrix. E.D. Hay, editor. Plenum Press. New York, New York, USA. 259-294.

2. Markwald, R.R., Fitzharris, T.P., Bank, H., and Bernanke, D.H. 1978. Structural analyses on the matrical organization of glycosaminoglycans in developing endocardial cushions. Dev. Biol. 62:292-316

3. Bernanke, D.H., and Markwald, R.R. 1979. Effects of hyaluronic acid on cardiac cushion tissue cells in collagen matrix cultures. Tex. Rep. Biol. Med. 39:271-285.

4. Camenisch, T.D., et al. 2000. Disruption of hyaluronan synthase- 2 abrogates normal cardiac morphogenesis and hyaluronan-mediated transformation of epithelium to mesenchyme. J. Clin. Invest. 106:349-360.

5. Weigel, P.H., Hascall, V.C., and Tammi, M. 1997. Hyaluronan synthases. J. Biol. Chem. 272:13997-14000 6. Spicer, A.P., and McDonald, J.A. 1998. Eukaryotic hyaluronan synthases. Glycoforum: science of byaluronan today. http://www.glycoforum.gr. jp/science/hyaluronan/HA07/HA07E.html.

7. DeAngelis, P.L., Papaconstantinou, J., and Weigel, P.H. 1993. Molecular cloning, identification, and sequence of the hyaluronan synthase gene from group A Streptococcus pyogenes. J. Biol. Chem. 268:19181-19184.

8. DeAngelis, P.L., and Weigel, P.H. 1994. Immunochemical confirmation of the primary structure of streptoccocal hyaluronan synthase and synthesis of high molecular weight product by the recombinant enzyme. Biochemistry. 33:9033-9039.

9. Toole, B.P. 1998. Hyaluronan in morphogenesis and tissue remodeling. Glycoforum: science of byaluronan today. http://www.glycoforum.gr. $\mathrm{jp} /$ science/hyaluronan/HA08/HA08E.html.

10. Toole, B.P. 2000. Hyaluronan. In Proteoglycans: structure, biology and molecular interactions. R.V Iozzo, editor. Marcel Dekker. New York, New York, USA. 61-92.

11. Oliferenko, S., Kaverina, I., Small, J.V., and Huber, L.A. 2000. Hyaluronic acid binding to CD44 acti- vates Rac1 and induces lamellipodia outgrowth. Cell Biol. 148:1159-1164.

12. Bourguignon, L.Y., Zhu, H., Shao, L., and Chen, Y.W. 2000. CD44 interaction with Tiam 1 promotes Rac1 signaling and hyaluronic acid-mediated breast tumor cell migration. J. Biol. Chem 275:1829-1838.

13. Evanko, S.P., Angello, J.C., and Wight, T.N. 1999. Formation of hyaluronan- and versican-rich pericellular matrix is required for proliferation and migration of vascular smooth muscle cells. Arterioscler. Thromb. Vasc. Biol. 19:1004-1013.

14. Yu, Q., and Stamenkovic, I. 1999. Localization of matrix metalloproteinase 9 (MMP-9) to the cell surface provides a mechanism for CD44-mediated tumor invasion. Genes Dev. 13:35-48.

15. Kosaki, R., Watanabe, K., and Yamaguchi, Y. 1999. Overproduction of hyaluronan by expression of the hyaluronan synthase has 2 enhances anchorage-independent growth and tumorigenicity. Cancer Res. 59:1141-1145.

16. Peterson, R.M., Yu, Q., Stamenkovic, I., and Toole, B.P. 2000. Perturbation of hyaluronan interactions by soluble CD44 inhibits growth of murine mammary carcinoma cells in ascites. Am. J. Pathol. 156:2159-2167.

17. Kamikura, D.M., Khoury, H., Maroun, C., Naujokas, M.A., and Park, M. 2000. Enhanced transformation by a plasma membrane-associated Met oncoprotein: activation of a phosphoinositide 3 '-kinasedependent autocrine loop involving hyaluronic acid and CD44. Mol. Cell. Biol. 20:3482-3496.

18. Fitzgerald, K.A., Bowie, A.G., Skeffington, B.S. and O'Neill, L.A. 2000. Ras, protein kinase C, and I $\kappa \mathrm{B}$ kinases 1 and 2 are downstream effectors of CD44 during the activation of NF- $\mathrm{KB}$ by hyaluronic acid fragments in T-24 carcinoma cells. J. Immunol. 164:2053-2063. 